\title{
A comparison of local anaesthetics for venepuncture
}

\author{
J Arrowsmith, C Campbell
}

\begin{abstract}
Aim-To compare the effectiveness of EMLA cream and Ametop gel in providing analgesia for venous cannulation. Methods-Single blind study in 120 children.

Results-Both anaesthetic agents produced adequate analgesia. However, Ametop gel was more effective, with a statistically significant difference in the pain scores of the two groups $(p<0.05)$. (Arch Dis Child 2000;82:309-310)
\end{abstract}

Keywords: topical anaesthetic; venepuncture

Topical local anaesthetic agents are proven to reduce the distress caused to children during venepuncture and venous cannulation. ${ }^{1}$ EMLA (eutectic mixture of local anaesthetics) has been available for a number of years and requires an application time of at least 60 minutes. $^{2}$ Ametop gel $(4 \% \mathrm{w} / \mathrm{w}$ amethocaine base preparation), a more recent preparation, has been shown to have a faster onset of maximum anaesthesia of $30-45$ minutes. $^{2}$

Previous comparative studies of the quality of anaesthesia have produced conflicting results. When both preparations were applied to adults for the recommended time for maximum anaesthesia, there was no statistical difference between the two preparations. ${ }^{3}$ However, when applied to children, for a time shorter than that recommended for EMLA, Ametop gel appeared to provide superior anaesthesia. ${ }^{4} 5$

Table 1 Application and cannulation details

\begin{tabular}{lll}
\hline & Ametop & EMLA \\
\hline Age (y) & $6.8(4.0)$ & $8.0(4.0)$ \\
Application time (h) & $2.04(1.0)$ & $1.93(1.0)$ \\
Time to venous cannulation (h) & $2.25(1.1)$ & $2.10(1.1)$ \\
Gauge of cannula & 20.7 & 21 \\
Number of attempts at cannulation & 1.9 & 1.2
\end{tabular}

Welsh Centre for Burns and Plastic Surgery, Morriston Hospital, Swansea SA6 6NL, Wales, UK J Arrowsmith

C Campbell

Correspondence to: Dr J Arrowsmith, Department of Plastic Surgery, Leicester Royal Infirmary, Leicester LE1 5WW, UK

Accepted 19 January 2000

Table 2 Behavioural distress scale results

\begin{tabular}{lll}
\hline & \multicolumn{2}{l}{ Number of children } \\
\cline { 2 - 3 } Behavioural score & Ametop & EMLA \\
\hline No response & 32 & 20 \\
Mild facial grimace & 11 & 13 \\
Verbal response & 6 & 10 \\
Crying & 5 & 10 \\
Withdrawal of hand & 6 & 7 \\
\hline
\end{tabular}

$\star_{\mathrm{p}}<0.05$, Mann-Whitney test.

\section{Methods}

After obtaining ethics committee approval and informed parental verbal consent, 120 children undergoing a variety of routine and emergency procedures were studied.

Sixty boys and 60 girls, aged from 1 to 15 years old, were randomly allocated to receive either EMLA cream or Ametop gel. The preparations were applied to the dorsum of the hands and/or the feet and covered with an occlusive dressing. When the child was scheduled to go to theatre, the preparation was removed and both the duration of application and any local reaction of the skin noted. Either the paediatric nurse or the anaesthetist, who were blinded to the topical anaesthetic used, assessed the pain experienced by the child during venous cannulation using a five point behavioural scale.

\section{Results}

The EMLA and Ametop groups were well matched. Table 1 shows the times of application, time to cannulation, and number of attempts at cannulation. These results were not significantly different, using the Yates corrected $\chi^{2}$ test.

EMLA produced significantly more blanching $(p<0.0001)$ and Ametop significantly more erythema $(p<0.018)$ at the site of application. There were no cases of hypersensitivity to either preparation.

The observed pain scores were significantly different between the two groups, the Ametop gel group exhibiting less response to cannulation than the EMLA group ( $<<0.05$; table 2). Excluding children who had more than one attempt at cannulation or children whose cannulation occurred later than four hours after application of the EMLA or five hours after application of the Ametop did not alter this result. These data were analysed using the Mann-Whitney test.

One boy experienced conjunctival irritation caused by rubbing his eye shortly after the Ametop gel had been removed from his hand. On review by an ophthalmologist, no corneal damage was identified and the conjunctivitis settled with conservative treatment.

\section{Discussion}

The results of this study show that both topical anaesthetic preparations provide effective anaesthesia for venous cannulation in children.

EMLA cream produced more blanching of the skin and Ametop gel more erythema owing to the different actions of the anaesthetic agents, the lignocaine and prilocaine in EMLA cream being predominantly vasoconstrictive and amethocaine a vasodilator. The difference 
in local skin reaction did not, however, appear to influence the number of attempts needed for successful venous cannulation.

In this study, $72 \%$ of children in the Ametop group exhibited either no response or a mild facial grimace on cannulation compared with $55 \%$ of children in the EMLA group. Overall the children in the Ametop group showed significantly less pain than the EMLA group.

The results of this study confirm previous reports ${ }^{34}$ that Ametop gel provides more effective anaesthesia for venous cannulation in a significantly higher proportion of children than does EMLA cream.
1 Arts SE, Abu-Saad HH, Champion GD, Crawford MR, Fisher RJ, Juniper KH, Ziegler JB. Age-related response to lidocaine-prilocaine (EMLA) emulsion and the effect of music distraction on the pain of intravenous cannulation. Pediatrics 1994;93:797-801

2 Freeman JA, Doyle E, Tee Im NG, Morton NS. Topical anaesthesia of the skin: a review. Paediatr Anaesth 1993;3:129-38

3 Molodecka J, Stenhouse C, Jones JM, Tomlinson A. Comparison of percutaneous anaesthesia for venous cannulation after topical application of either amethocaine or EMLA cream. Br 7 Anaesth 1994;72:174-6.

4 Woolfson AD, McCafferty DF, Boston V. Clinical experiences with a novel percutaneous amethocaine preparation: prevention of pain due to venepuncture in children. $\mathrm{Br} F$ Clin Pharmacol 1990;30:273-9.

5 Lawson RA, Smart NG, Gudgeon AC, Morton NS. Evaluation of an amethocaine gel preparation for percutaneous analgesia before venous cannulation in children. $\mathrm{Br} F$ Anaesth 1995;75:282-5.

\section{Sex preference}

When you were an adolescent would you have opted to have your genitalia examined by a doctor of your own sex or of the opposite sex? It seems to me that most of us would have preferred a doctor of our own sex simply to save ourselves from potential embarrassment. A study in Kansas City, however, has suggested the opposite (Christopher J Van Ness and Daryl A Lynch. Archives of Pediatrics and Adolescent Medicine. 2000;154:49-53).

They asked 81 male adolescents (aged 10-18) attending a hospital adolescent clinic to complete a questionnaire and 67 did so. More of the respondents indicated a preference for female doctors than for male doctors, both for general examination $(51 \% v 39 \%)$ and for genital examination $(49 \%$ v 39\%). [The American Cancer Society recommends that male adolescents should examine their own genitalia monthly and be examined by a doctor annually as a screen for testicular cancer.] The preference for a woman doctor was greater in African American respondents, almost two thirds of whom had been raised by a single female parent, relative, or guardian.

These authors warn against accepting their findings at face value because of small numbers and a low response rate. Do people, especially adolescents, always answer questionnaires in a perfectly straightforward and truthful manner? Could adolescent iconoclasm and tongue-in-cheek humour have an effect? Or is that just too cynical?

ARCHIVIST 